\title{
Steganographic Generative Adversarial Networks
}

\author{
Denis Volkhonskiy ${ }^{1}$, Ivan Nazarov ${ }^{1}$, Evgeny Burnaev ${ }^{1}$ \\ ${ }^{1}$ Skolkovo Institute of Science and Technology \\ Nobel street, 3, Moscow, Moskovskaya oblast', Russia \\ e-mail: e.burnaev@skoltech.ru
}

\begin{abstract}
Steganography is collection of methods to hide secret information ("payload") within non-secret information "container"). Its counterpart, Steganalysis, is the practice of determining if a message contains a hidden payload, and recovering it if possible. Presence of hidden payloads is typically detected by a binary classifier. In the present study, we propose a new model for generating image-like containers based on Deep Convolutional Generative Adversarial Networks (DCGAN). This approach allows to generate more setganalysis-secure message embedding using standard steganography algorithms. Experiment results demonstrate that the new model successfully deceives the steganography analyzer, and for this reason, can be used in steganographic applications.
\end{abstract}

Keywords: generative adversarial networks, steganography, security

\section{INTRODUCTION}

Recent years have seen significant advances in estimation methods and application of deep generative models. There are two major general frameworks for learning deep generative models: Variational Autoencoders (VAEs), [17], and Generative Adversarial Networks (GANs), [7]. The recent work of Hu et al. [11] develops a unifying framework, which establishes strong connections of these approaches to Adversarial Domain Adaptation (ADA), [5].

GANs have achieved impressive results in semi-supervised learning, [16], and image-to-image translation, [13]. In [7] the success of GANs framework was illustrated on the problem of image generation. A more recent paper [29] proposed as set of constraints on the architecture of convolutional GANs and showed that thus restricted deep convolutional GANs (DCGANs) are capable of learning a hierarchy of representations from object parts to scenes, which are sufficiently robust to transfer across domains.

In this study we apply the DCGAN framework to the domain of steganography, i.e. practical approaches to concealing information (payload) within another piece of information (stego-container). In particular, we train a generator, images produced by which are less susceptible to steganographic analysis compared to the original images used as stego-containers. At the same time, we require that the induced distribution of the synthetic images approximate well the distribution of the real images in the dataset.

Thus we train a generative model for image stego-containers by confronting it with two deep convolutional adversaries: a discriminator network, which regularizes the output to look like samples from the real dataset, and a steganographic analyzer, which aims at detecting if an image conceals a hidden message. The presence of two regularizers in the generator's objective resembles the recently proposed multi-target GAN framework [2].

\section{STEGANOGRAPHY}

Steganography is a set of algorithms for concealing information in inconspicuous-looking communication and a collection of methods to detect and recover the hidden message from suspicious media (Steganalysis). In steganography the information to be hidden, the payload, is embedded by an algorithm inside a cover medium, the stego-container. The key drawback is that steganography offers security through obscurity: an embedded message is sent in the hope that a third party won't detect or discover it. This makes pure steganography impractical without cryptography, which deals with secure communication over an insecure channel: the message is scrambled and authenticated with some keyed algorithm before being concealed in a cover medium, [1, 21]. In this respect steganography serves as a layer of weak security by adding a cyphertext detection and extraction step: encrypted data has much higher entropy than the regular data. Besides information protection and covert communication, steganography is useful for watermarking in digital rights management and user identification.

The simplest and most popular algorithm of unkeyed stego-embedding is called the Least Significant Bit (LSB) matching. The main idea is to take the binary representation of a secret message, pad it, and store it in a stego-container by overwriting the LSB of each byte within. The cover-media used for the LSB embedding must be resilient with respect to 
bit-level augmentation. In the case of images the least significant bits of each colour channel of each pixel in the given image are used to hide the payload.

The perturbations introduced by the LSB algorithm do not preserve marginal or joint colour statistics, which despite being imperceptible to a human observer, simplify detection of the hidden payload with machine learning or statistical models. A modification of this method, which addresses this issue to some extent, is the so-called \pm 1 -embedding: each bit of the message is hidden by randomly adding or subtracting 1 from a pixel's colour channel so that the last bit matches it.

More sophisticated steganographic schemes modify the digital media adaptively. The key idea is to constrain the embedding to regions of high local entropy, e.g. complex textures or noise. Each pixel is assigned an embedding cost and the embedding locations are picked in such a way as to minimize the distortion function

$$
D(I, \hat{I})=\sum_{i, j} \rho_{i j}\left(I, \hat{I}_{i j}\right)
$$

where $I$ is the cover image, $\hat{I}$ is the stego-embedding, and $\rho_{i j}\left(I, \hat{I}_{i j}\right)$ is the bounded cost of altering a pixel $(i, j)$ in the cover-image $I$. The embedding itself is performed by coding methods such as Syndrome-Trellis Codes (STC) [3], which are essentially binary linear convolutional codes represented by parity-check matrix. The state-of-the-art content-adaptive stego-embedding algorithms include HUGO [24], which computes the embedding costs based on Subtractive Pixel Adjacency Matrix (SPAM) features [23]; WOW [9] and S-UNIWARD [10], which use directional wavelet filters to weigh and pick regions with high entropy, but implement different embedding cost functional.

\subsection{Steganalysis}

The simplest approach to steganalysis is based on special feature extractors, e.g. SPAM [23], SRM [4], combined with traditional machine learning models, such as support vector classifiers, decision trees, classifier ensembles, et c. With the recent overwhelming success of deep learning, specifically in the image classification and generation domain, newer approaches based on deep Convolutional Neural Networks (deep CNN) are gaining popularity. For example, in [27] it is shown that deep CNN with Gaussian activation functions achieve competitive performance with hand-crafted features, and in [25] it is demonstrated that even shallow CNN are able to outperform the usual ML based stego-analysis techniques in terms of the detection accuracy.

In this paper we consider steganographic embedding of random bit messages into specifically crafted images using the \pm 1 -embedding algorithm. The security of the stego-containers is tested against a class of deep convolutional neural network stego-analyzers, which try to distinguish images with hidden data from the empty ones.

\subsection{Problem Statement}

The total scheme of steganography and steganalysis is presented at Fig. 1

- Usually all images are attacked by a stegoanalyser (Eve);

- Alice (Steganography algorithm) tries to deceive Eve;

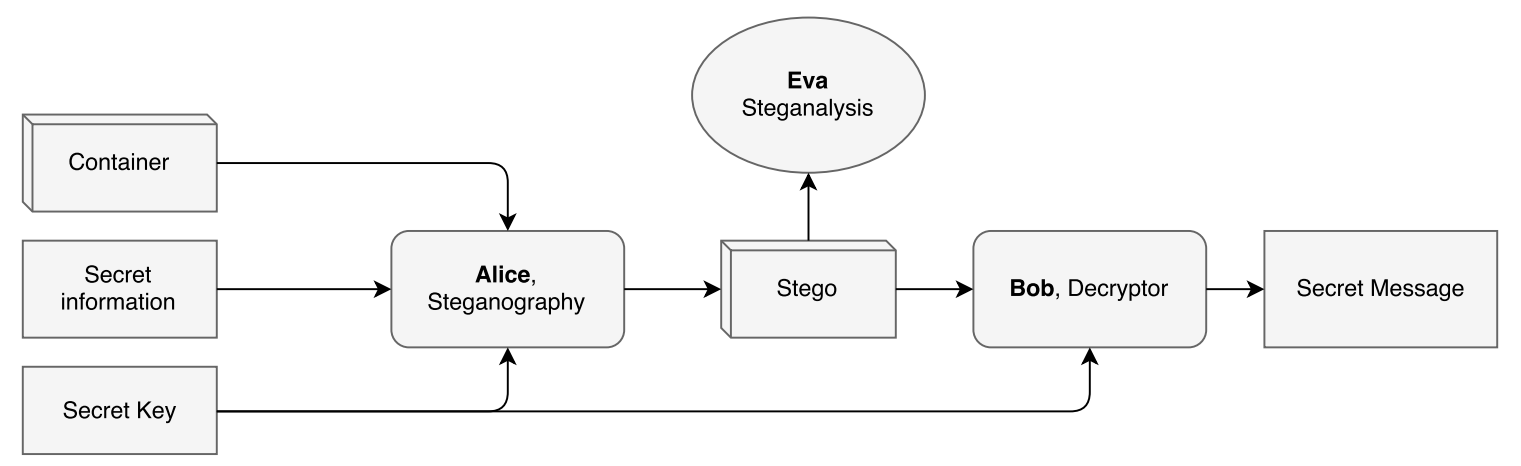

Figure 1: Complete scheme of steganography and steganalysis

The disadvantage of the standard steganography approach is inadaptability of the containers and algorithms for Eve. By this we mean that containers don't adopt (and even know) for type of Eve. The goal for this work is to create adaptive containers generator and steganography new method. 


\subsection{Tasks for the research}

We would like to obtain adaptability of the containers to the given Steganalysis in order to deceive it. We set the following tasks for the current work:

1. Adaptive containers generation.

- Create a model for image containers generation, that can be used with any Steganography algorithm;

- Using of these generated containers should deceive Eve (steganalysis);

- Containers should be adaptive to any type on Eve.

2. New Steganography method:

- Create a model for adaptive generation of images with hidden information inside;

- Test the quality of encryption-decryption on MNIST and CIFAR-10 datasets

The difference between these two tasks is the following.

In the first model, we would like to build a generator of empty containers (images). This images could be used with any Steganography algorithm.

In the second model, we would like to generate not only empty images, but to encode the information to them for further extraction. In other words, we would like to obtain an analogy of visual markers (such as QR-codes).

\section{GENERATIVE ADVERSARIAL NETWORKS}

Generative Adversarial Networks (GANs) training, [7], is a powerful framework for estimating generative models in unsupervised learning setting by way of a two-player minimax game. The generator player attempts to mimic the true data distribution $p_{\text {data }}(x)$ by learning a transformation function $z \mapsto G_{\theta}(z)$ of random input values $z$, drawn from a tractable distribution $p_{z}$. The generator receives feedback from the discriminator $D_{\phi}$, which strives to distinguish synthetic, "fake" samples $x=G(z), z \sim p_{z}$, from genuine, "real" samples $x \sim p_{\text {data }}$.

In the original formulation, [7], the learning process of the generator $(G)$ and the discriminator $(D)$ consists of searching for a saddle point solution of the following optimization problem

$$
\min _{\theta} \max _{\phi} \mathscr{L}(\theta, \phi)=\mathbb{E}_{x \sim p_{\text {data }}}[\log D(x ; \phi)]+\mathbb{E}_{z \sim p_{z}}[\log (1-D(G(z ; \theta) ; \phi))],
$$

where $D(x ; \phi)$ is the probability output by the player $D$ that $x$ is a real sample rather then synthetic, and $G(z ; \theta)$ is the generated sample. In a typical application the ground truth data distribution is provided implicitly through its finite sample approximation on the dataset $\mathscr{D}=\left(x_{i}\right)_{i=1}^{m}$. Furthermore, the expectations in the are approximated by sample averages over randomly drawn mini-batches of $\left(G\left(z_{i} ; \theta\right)\right)_{i=1}^{B}$ for $\left(z_{i}\right)_{i=1}^{B} \sim p_{z}$ i.i.d. and $\left(x_{i}\right)_{i=1}^{B}$ sampled without replacement from the training set.

Despite many advantages, such as generation with a single forward pass and asymptotically consistent data distribution estimation, the major disadvantage of GANs is that training them requires finding a Nash (best response) equilibrium, [6]. Furthermore, since for deep networks $G(\cdot ; \theta)$ and $D(\cdot ; \phi)$ the objective is non-convex w.r.t. the parameters $\phi$ and $\theta$, the order of min and max in (1) matters. Therefore [7] propose to solve the problem by iteratively alternating between SG maximization and minimization steps, but giving optimizational advantage to the discriminator player. The idea is to make several gradient ascent steps on $\mathscr{L}(\theta, \phi)$ w.r.t. $\phi$, before a single gradient descent step w.r.t. $\theta$.

By giving advantage to the discriminator, the proposed approach attempts to approximate what in fact is the generator's true objective: $\theta \mapsto \max _{\phi} \mathscr{L}(\theta, \phi)$. However, since in practice may GANs are trained with alternating single-step updates, [22] propose and justify a simpler joint single-step gradient method: the SGD update moves along the joint direction $\left(\nabla_{\theta} \mathscr{L},-\nabla_{\phi} \mathscr{L}\right)$ obtained via a single back-propagation step. However, there is no consensus as to what the best training scheme for solving (1] is, [6].

GAN training is also complicated by the different regimes the networks undergo in the process. For instance, during early stage of training the discriminator is prone to becoming excessively powerful, which makes the last term of (1) provide weak feedback to the generator. In [7] authors suggest to use $\mathscr{L}_{\text {Gen }}(\theta ; \phi)=-\mathbb{E}_{z \sim p_{z}}[\log D(G(z ; \theta) ; \phi)]$ as the minimization objective of the generator, and to set $-\mathscr{L}(\theta, \phi)$ as the discriminator's loss, $\mathscr{L}_{\text {Dis }}(\phi ; \theta)$, to be minimized. In spite of changing the loss and making the game no longer zero-sum, this heuristic leads to the same saddle point as demonstrated in [22].

In fig. 2] we depict a sample of synthetic images of a freshly trained DCGAN on the Celebrities dataset [20]. The images indeed look realistic, albeit with occasional artifacts. 


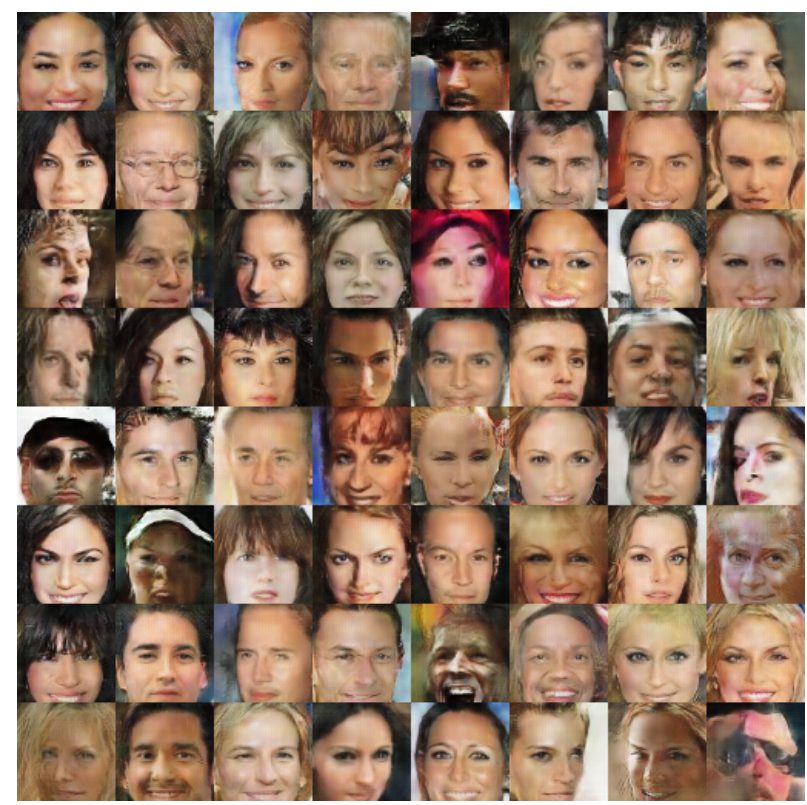

Figure 2: Sample synthetic images generated by DCGAN

\section{STEGANOGRAPHIC GENERATIVE ADVERSARIAL NETWORKS}

\subsection{Model description}

Let $\mathscr{I}=[-1,1]^{H \times W \times 3}$ be the space of images with dimensions $H \times W$ and RGB channel saturation values between -1 and 1 . Let $p_{z}$ be a uniform distribution on $\mathscr{Z}=[-1,1]^{d_{z}}$, and $p_{\text {data }}$ be the distribution of reference images on a subset of $\mathscr{I}$. Finally, the set of messages $\mathscr{M}$ is given by $\{0,1\}^{d_{M}}, d_{M} \leq H W$, and by $S_{m}: \mathscr{I} \mapsto \mathscr{I}$ we denote the LSB embedding of the message $m \in \mathscr{M}$ in the cover image $x \in \mathscr{I}$.

We introduce Steganographic Generative Adversarial Networks model (SGAN), which is a zero-sum game between two players: a generator network $G: \mathscr{Z} \mapsto \mathscr{I}$, which tries to mimic $p_{\text {data }}$, and an adversary consisting of two parts

- a discriminator network $D: \mathscr{I} \mapsto[0,1]$, which distinguishes synthetic images $x=G(z), z \sim p_{z}$, from real $x \sim p_{\text {data }}$;

- a steganalyzer network $A: \mathscr{I} \mapsto[0,1]$, which tries to separate cover images $S_{m}(x)$ with payload $m \in \mathscr{M}$, from empty images $x, x \sim Q$, for some image distribution $Q$ on $\mathscr{I}$.

The value function of the game is fig. 3

$$
\begin{gathered}
\mathscr{L}(\theta, \phi, \psi)=\alpha \mathscr{L}_{\text {Dis }}(\phi ; \theta)+(1-\alpha) \mathscr{L}_{\operatorname{San}}(\psi ; \theta) \rightarrow \min _{\theta} \max _{\phi, \psi}, \\
\mathscr{L}_{\text {Dis }}(\phi ; \theta)=\mathbb{E}_{x \sim p_{\text {data }}}[\log D(x ; \phi)]+\mathbb{E}_{z \sim p_{z}}[\log (1-D(G(z ; \theta) ; \phi))], \\
\mathscr{L}_{\text {San }}(\psi ; \theta)=\mathbb{E}_{z \sim p_{z}}\left[\mathbb{E}_{m}\left[\log A\left(S_{m}(G(z ; \theta)) ; \psi\right)\right]\right]+\mathbb{E}_{z \sim p_{z}}[\log (1-A(G(z ; \theta) ; \psi))] .
\end{gathered}
$$

Here the discriminator and the steganalyzer maximize the likelihood $\mathscr{L}_{\text {Dis }}$ and $\mathscr{L}_{\text {San }}$, respectively, while the generator minimizes the convex combination of likelihoods of $D$ and $A$ in $(2)$. The mixing parameter $\alpha \in(0,1)$ controls the trade-off between the importance of realism of generated images and their quality as containers against the steganalysis. Analysis of preliminary experimental results showed that for $\alpha \leq 0.5$ the generated fails to approximate the distribution of the reference images.

This model resembles the recently proposed multi-target GANs framework, [2], which pits the generator against multiple discriminators: a boosted ensemble, a mean aggregated combination of discriminators, or discriminators with adaptively adjustable power. The main idea of the paper naturally suggests that additional discriminators can be used for regularization, adaptation of the generator's output to other domains, or endowing the synthetic samples with certain required properties.

The we propose to train the model with the joint simultaneous gradient update scheme proposed in [22]: jointly backpropagate through the networks and update 
- for $D$ with $\phi \leftarrow \phi+\gamma_{D} \nabla_{\phi} \mathscr{L}_{\text {Dis }}$

- for $A$ with $\psi \leftarrow \psi+\gamma_{A} \nabla_{\psi} \mathscr{L}_{\text {San }}$;

- for $G$ with $\theta \leftarrow \theta-\gamma_{G} \nabla_{\theta} \mathscr{L}$, where $\mathscr{L}$ is as in (2).

The expectations are substituted by the empirical averages over the joint mini-batch of images $x \sim p_{\text {data }}$, noise $z \sim p_{z}$, and messages $m \sim\{0,1\}^{d_{M}}$.

It is expected that the SGAN game would, for suitable class of deep CNN and an appropriate training schedule, yield an equilibrium in which the generator produces realistic images capable of concealing messages embedded by LSB against a deep convolutional steganalyzer. As is in the case of the original formulation [7], if the players were not confined to the class of deep convolutional networks, the optimal steganalyzer would have been given by the ratio estimator between the distribution of the images $q$, induced by $G(z ; \theta)$ for $z \sim p_{z}$, and the distribution $q_{s}$, implicitly defined as the distribution of $S_{m}(x)$ over $x \sim q$ and $m \sim\{0,1\}^{d_{M}}$. We expect that the optimal generator would induce a distribution, the variates from which would have uniformly random least significant bit in the colour data of each pixel, since for a random $m$ the stego-embedding $S_{m}(x)$ alters $x$ only on the scale of $2^{-7}$, and the result is, essentially, a random bit.

\subsection{Challenges}

One of the challenges in the proposed model is the fact that any stego-embedding algorithm introduces a distortion to the cover medium, which is generally a non-differentiable perturbation as a function of the data in the medium. For instance, the LSB matching modifies the colour channel data of the pixels independently, and thus can be represented as a residual-like transformation of each colour channel value: $S_{m}(x)=x+\delta_{m}(x)$, for any message bit $m \in\{0,1\}$ and $x \in[-1,+1]$, where $\delta_{m}(x) \in\{0, \pm \varepsilon\}, \varepsilon=2^{-7}$, is the distortion. The $\delta_{m}(x)$ is a random function given by

$$
\delta_{m}(x)=\xi \mathbf{p}_{m}\left((x+1) \varepsilon^{-1}\right),
$$

where $\mathbf{p}_{m}(z)$ is 0 when $\exists k \in \mathbb{Z}: z-m \in[2 k, 2 k+1)$, i.e. the LSB of the integer part of $z$ matches $m$, and 1 otherwise. The value $\xi$ is $+\varepsilon$ if $m=1$ and $x \in[-1,-1+\varepsilon)$, and $-\varepsilon$ if $m=0$ and $x \in(+1-\varepsilon,+1]$, but otherwise an independent random variable $\pm \varepsilon$, which is the addition / subtraction mask in the LSB algorithm.

The key observation is that for fixed $m$ and $\xi$ this perturbation is fully determined by $\mathbf{p}_{m}(z)$, which is constant on the intervals $(k, k+1)$ with $k \in\{0 \ldots 255\}$. While this function is non-differentiable at finitely many points, where the $0-1$ switching occurs, everywhere else in $(0,256)$ it is constant and has zero derivative w.r.t. $z$. Thus for fixed $m$ and $\xi$ the distortion $\delta_{m}(x)$ has derivative zero almost for every $x \in(-1,1)$. In light of this heuristic argument, the following procedure for stego-embedding can be used while training: during the forward pass the embedding $x \mapsto S_{m}(x)$ is exact, but during the back propagation the embedding response is approximate by an identity function $S_{m}(x) \approx x$.

The main drawback of this "linear" approximation is that it provides essentially no gradient feedback to the generator and acts as if nothing was embedded. Thus, although being almost correct it is ill suited for the purpose of learning stego-secure cover entities by design. Therefore we propose another approach: for a fixed message $m \in\{0,1\}$ and noise $\xi \in\{ \pm 1\}$ approximate the LSB embedding layer by a differentiable transformation. To this end we substitute the mismatch indicator in (5) by a sine waveform with nonlinear gain at extremes of its range:

$$
\mathbf{p}_{m}(z) \approx \mathbf{s}_{\beta}(z ; m)=\sigma(\beta \sin (m-z) \pi),
$$

where $\beta>0$ determines the fidelity of the approximation and $\sigma(a)$ is the Sigmoid function, $a \mapsto\left(1+e^{-a}\right)^{-1}$. The derivative of the Sigmoid function is $a \mapsto \sigma(a)(1-\sigma(a))$, and therefore this approximation is not computationally too demanding and provides accurate gradient feedback at the jump points of $\mathbf{p}_{m}(z)$.

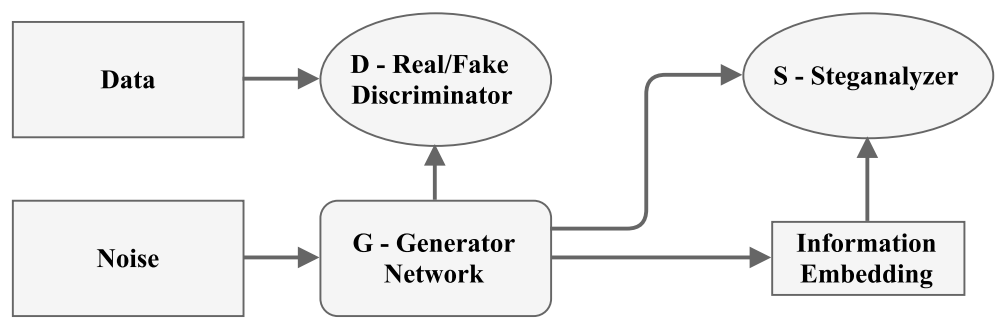

Figure 3: SGAN information flow diagram 


\subsection{Training process}

Stochastic mini-batch Gradient descent update rules for components of SGAN are listed below:

- for $D$ the rule is $\theta_{D} \leftarrow \theta_{D}+\gamma_{D} \nabla_{G} L$ with

$$
\nabla_{G} L=\frac{\partial}{\partial \theta_{D}}\left\{\mathbb{E}_{x \sim p_{\text {data }}(x)}\left[\log D\left(x, \theta_{D}\right)\right]+\mathbb{E}_{z \sim p_{\text {noise }}(z)}\left[\log \left(1-D\left(G\left(z, \theta_{G}\right), \theta_{D}\right)\right)\right]\right\} ;
$$

- for $S$ (it is updated similarly to $D$ ): $\theta_{S} \leftarrow \theta_{S}+\gamma_{S} \nabla_{S} L$ where

$$
\nabla_{S} L=\frac{\partial}{\partial \theta_{S}} \mathbb{E}_{z \sim p_{\text {noise }}(z)}\left[\log S\left(\operatorname{Stego}\left(G\left(z, \theta_{G}\right)\right), \theta_{S}\right)+\log \left(1-S\left(G\left(z, \theta_{G}\right), \theta_{S}\right)\right)\right] ;
$$

- for the generator $G$ : $\theta_{G} \leftarrow \theta_{G}-\gamma_{G} \nabla_{G} L$ with $\nabla_{G} L$ given by

$$
\begin{aligned}
\nabla_{G} L & =\frac{\partial}{\partial \theta_{G}} \alpha \mathbb{E}_{z \sim p_{\text {noise }}(z)}\left[\log \left(1-D\left(G\left(z, \theta_{G}\right), \theta_{D}\right)\right)\right]+\frac{\partial}{\partial \theta_{G}}(1-\alpha) \mathbb{E}_{z \sim p_{\text {noise }}(z)}\left[\log \left(S\left(\operatorname{Stego}\left(G\left(z, \theta_{G}\right), \theta_{S}\right)\right)\right)\right] \\
& +\frac{\partial}{\partial \theta_{G}}(1-\alpha) \mathbb{E}_{z \sim p_{\text {noise }}(z)}\left[\log \left(1-S\left(G\left(z, \theta_{G}\right), \theta_{S}\right)\right)\right] .
\end{aligned}
$$

The main distinction from the GAN model is that we update $G$ in order to maximize not only the error of $D$, but to maximize the error of the linear combination of the classifiers $D$ and $S$.

\section{STEGANOGRAPHIC ENCRYPTION GENERATIVE ADVERSARIAL NETWORKS}

\subsection{Model Description}

Steganographic Encryption Generative Adversarial Networks (SEGAN) model was constructed for information encryption/ description purposes. It consists of

- Alice - A generator network: produce realistic images, that contains hidden information.

- Input: Secret Key (binary), Secret Message (binary), Class for generation (y), noise

- Output: Image with hidden secret message inside

- Bob - A decryption network: extract hidden message from the image.

- Input: Image, Secret Key, Class of the image (y)

- Output: Secret hidden message

- Discriminator - A discriminator, that tries to detect whether the image is real or generated.

- Input: Image, Class of the image (y)

- Output: 0/1 - generated/real class

The full scheme is presented in Fig. 4. This model can be considered as a autoencoder with the high-dimensional hidden representation. In such case Discriminator is considered as such kind of regularization.

\subsection{Training process}

The training process can be represented as a usual GAN training process, but with some modification. Firstly we should define the loss functions for constructing the updating rules:

- Alice (as realistic image Generator) loss. Standard GANs generator loss.

$$
L_{A}=\mathbb{E}_{y} \mathbb{E}_{m \sim p_{\text {message }}} \mathbb{E}_{k \sim p_{\text {key }}} \mathbb{E}_{z \sim p_{\text {noise }}}\left[\log \left(1-C\left(\theta_{C},\left(\theta_{A}, m, k, z, y\right), y\right)\right) \mid y, m, k, z\right]
$$

- Alice-Bob encryption loss. Here we compute standard $l 2$ loss between the original message and reconstructed message.

$$
L_{A B}=\mathbb{E}_{y} \mathbb{E}_{m \sim p_{\text {message }}} \mathbb{E}_{k \sim p_{\text {key }}} \mathbb{E}_{z \sim p_{\text {noise }}} \mathbb{E}\left[\left(m-B\left(\theta_{B}, A\left(\theta_{A}, m, k, z, y\right), k, y\right)\right)^{2} \mid y, m, k, z\right] \rightarrow \min
$$




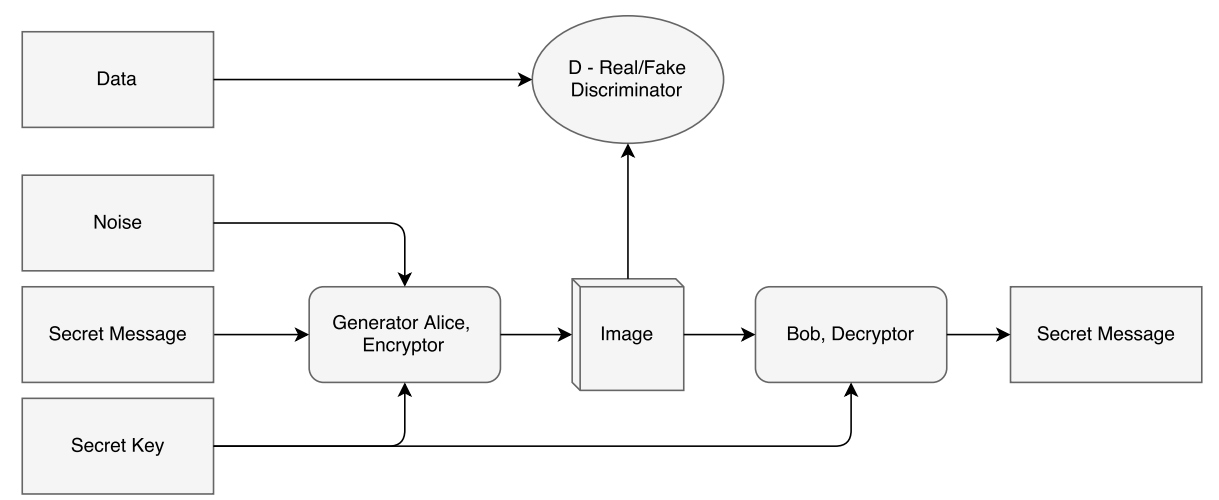

Figure 4: SEGAN information flow diagram

Input $: d_{k}-$ The dimension of a binary key

$d_{m}$ - The dimension of a binary message,

$d_{n}$ - The dimension of the input noise

for epoch in 1...n_epoch do

for minibatch from the data set do

Sample minibatch of $d_{n}$ noise samples $\left\{z_{1}, \ldots, z_{d_{n}}\right\}$

Sample minibatch of $d_{m}$ noise samples $\left\{m_{1}, \ldots, m_{d_{m}}\right\}$

Sample minibatch of $d_{k}$ noise samples $\left\{k_{1}, \ldots, k_{d_{k}}\right\}$

Update $A$ according to loss $L_{A}$

Update $C$ according to loss $L_{C}$

if epoch $>1$ then

| Update $A, B$ according to loss $L_{A B}$ end

end

end

Algorithm 1: Algorithm of training SEGAN

- Discriminator's loss. This is a usual GANs discriminator loss, witch is calculated as an average cross-entropy.

$$
\begin{aligned}
L_{C}= & \mathbb{E}_{y} \mathbb{E}_{x \sim p_{\text {data }}}\left[\log \left(C\left(\theta_{C}, x, y\right)\right) \mid y\right]- \\
& -\mathbb{E}_{y} \mathbb{E}_{m \sim p_{\text {message }}} \mathbb{E}_{k \sim p_{\text {key }}} \mathbb{E}_{z \sim p_{\text {noise }}}\left[\log \left(1-C\left(\theta_{C}, A\left(\theta_{A}, m, k, z, y\right), y\right)\right) \mid y, m, k, z\right] \rightarrow \min
\end{aligned}
$$

The total SEGAN train procedure is presented in Algorithm 1

\section{EXPERIMENTS WITH STEGANOGRAPHIC GENERATIVE ADVERSARIAL NETWORKS}

\subsection{Steganographic Vectors}

Before conducting extensive numerical experiments on the security of the LSB embedding in cover images produced by a SGAN trained generator, we run a simpler experiment as a proof-of-concept. Since the LSB matching embeds each bit of the message into a pixel independently from the its context, we study numerically the stego-security properties of SGAN generated 1-d vectors.

\subsubsection{Validation Protocol}

In the process of training SGAN for $T$ iterations we obtain a sequence of generators $\left(G^{t}\right)_{t=1}^{T}$ where $G^{t}(\cdot)=G\left(\cdot ; \theta_{t}\right)$ and $\theta_{t}$ are the parameters of the generator after $t$ minibatch SGD updates. We use the following empirical validation protocol for the generator after the $t$-th iteration:

1. Draw a sample $\mathscr{S}^{t}=\left(x_{i}, y_{i}\right)_{i=1}^{M}$, i.e. for $i=1, \ldots M$ 
(a) independently draw $y_{i} \sim\{0,1\}$ and $z_{i} \sim p_{z}$;

(b) get a message $m_{i} \in \mathscr{M}=\{0,1\}^{d_{M}}$;

(c) synthesize a cover entity $x_{i}^{*}=G^{t}\left(z_{i}\right)$ and set $x_{i}= \begin{cases}S_{m_{i}}\left(x_{i}^{*}\right), & \text { if } y_{i}=1, \\ x_{i}^{*}, & \text { if } y_{i}=0 .\end{cases}$

2. Assess the performance of an independent steganalyzer $A^{*}$ with $K$-fold cross validation on $\mathscr{S}^{t}$.

This sampling procedure ensures that the examples in the stego-sample $\mathscr{S}^{t}$ are independent and identically distributed.

We also control the diversity of the embedded messages $m_{i}$ through different message generation scenarios:

- Fixed: $m_{i}=m_{0}$, with $m_{0}$ picked once from $\mathscr{M}$;

- Pool (n): random $m_{i}$ from $\mathscr{M}_{0} \subset \mathscr{M},\left|\mathscr{M}_{0}\right|=n$;

- Arbitrary: random $m_{i}$ from $\mathscr{M}$.

Under the "Fixed" and "Pool (n)" variants $m_{0}$ and $\mathscr{M}_{0}$, respectively, are chosen independently for each sample $\mathscr{S}^{t}$ in the above outline. The motivation behind controlling the diversity stems from the idea that by comparing the optimal performance of $A^{*}$ under each scenario it is possible to verify if $S_{m}(G(z)) \equiv G(z)$ in distribution for independent $z \sim p_{z}$ and $m \sim \mathscr{M}$. In fact, with SGAN it is possible to train a generator, that induces an distribution, which is invariant under LSB embedding of messages from a specific, non-uniform distribution of messages $\mathscr{M}$ just by sampling from it during training.

We use this validation protocol for the experiments in sec. 6.1. Howeer, for the experiments with image generation ( sec. 6.2 we extend the protocol, since in this case we have a reference distribution $Q$ on $\mathscr{I}$, which the generator $G$ aims to replicate.

The extension is illustrated in fig. 5. Firstly, several independent real and synthetic training datasets are generated:

1. Draw a synthetic stego-sample $\mathscr{S}_{\mathrm{S}}$ as outlined above;

2. Generate similarly a real stego-sample $\mathscr{S}_{\mathrm{R}}$ by sampling $x_{i}^{*}$ from $Q$ (sample without replacement if $Q$ is an empirical distribution);

Secondly, on each of these datasets we train an independent steganalyzer $A^{*}$ and aggregate them with weighted majority voting to obtain the final pair of steganalyzers $A_{\mathrm{S}}^{*}$ and $A_{\mathrm{R}}^{*}$, where the former is based on synthetic training datasets and the latter - on real stego-samples. Finally, we independently generate several real and synthetic datasets as outlined above, and used them for cross-validation of the steganalyzers: each one is validated on both kinds of test samples to assess if and how well the learned features transfer across real / synthetic domains.

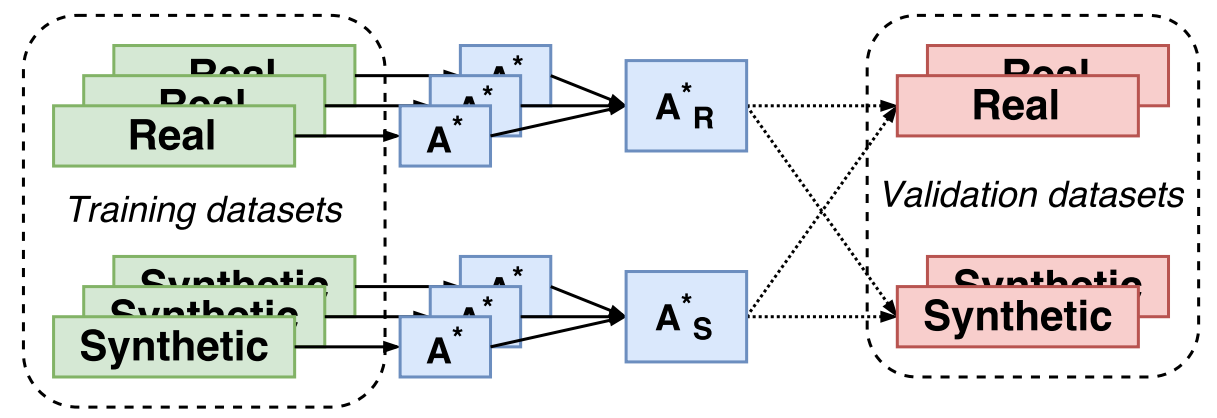

Figure 5: Extended validation protocol for experiments in sec. 6.1

\subsubsection{Details of the Experiment}

The generator $G$ is a $1-\mathrm{d}$ convolutional neural network $(\mathrm{CNN})$ that expands the input $z \sim \mathscr{N}_{d_{I}}\left(0, I_{d_{I}}\right)$ from $\mathbb{R}^{d_{I}}$ into $[-1,+1]^{d_{O}}$, where $d_{I}=4$ and $d_{O}=16$. The generator uses a series of 1-d transposed convolution layers with nonunit strides and the ReLU nonliearity, $a \mapsto \max \{a, 0\}$, to upsample the input noise into a intermediate $32 \times d_{O}$ state matrix, which is finally passed through a 1-d convolution layer with Tanh nonlinearity, $a \mapsto \frac{e^{a}-e^{-a}}{e^{a}+e^{-a}}$, to ensure $[-1,+1]^{d_{O}}$ output.

The steganalyzer $A$ is also a $1-\mathrm{d}$ CNN which takes a sample $x \in[-1,+1]^{d_{O}}$ and feeds the input through a set of 1-d convolution layers with Leaky ReLU, $a \mapsto \max \{\alpha a, a\}$ for $\alpha=\frac{1}{20}$ to get the $256 \times 4$ matrix of intermediate features. The matrix is passed through two fully connected layers with the Leaky ReLU and 512 units each. The final layer of $A$ outputs a single logit score, which reflects the degree to which the analyzer is "confident" that the sample $x$ contains a message 
hidden with the LSB embedding. This network $A$, as well as $G$, has a Batch Normalization (BN) layer, [12], before the nonlinearity, except for the output layers.

The losses are set-up as in (2) and (4), but without an explicit "discriminator" player. We simulate its feedback by imposing a total variation with $l_{2}$ loss on the generator's output:

$$
\mathscr{L}_{\text {Dis }}(\theta)=\left.\mathbb{E}_{z \sim p_{z}}\left[\sum_{i=1}^{d_{O}} C_{\mathrm{tv}}\left|x_{i}-x_{i-1}\right|+C_{\mathrm{1}_{2}} \frac{1}{2} x_{i}^{2}\right]\right|_{\substack{x=G(z ; \theta) \\ x_{0}=x_{d_{O}}}} .
$$

The relative weight $\alpha$ in the generator's loss is set to $\alpha=\frac{C_{\mathrm{san}}}{C_{\mathrm{san}}+C_{\mathrm{tv}}+C_{1_{2}}}$ for $C_{\mathrm{san}}=2, C_{\mathrm{tv}}=10^{-2}$, and $C_{\mathrm{1}_{2}}=10^{-1}$.

We train SGAN generator for $T=75 \cdot 10^{3}$ minibatch iterations of 256 pairs of noise and message vectors. We set $d_{M}=d_{O}$ and thus embed 1 bit of message in each element of the cover vector, achieving the flat vector analogue of image's 1 bit-per-pixel embedding density.

SGAN networks are trained with Adam, [14], with default parameter values and learning rates $2^{-14}$ and $2^{-18}$ for the steganalyzer $(A)$ and the generator $(G)$, respectively. We use different learning rates to make the generator adapt slowly to the steganalyzer's feedback, while allowing the analyzer to learn to detect hidden data in the generator's output more quickly, [8]. The training was carried out with the strategy proposed in [7]: 4 updates of $A$ per 1 update of $G$. The scale parameter $\beta$ of (6) was set to 8 .

We run the validation protocol with the sample size $M=2^{16}$ and the number of folds $K$ set to 7 . In this setting we validate two different independent steganalyzers $A^{*}$ : a Random Forest classifier ensemble with 101 trees and a 1- $d C N N$ similar to the network used during SGAN training. The only difference of the CNN $A^{*}$ is that it yields two values and passes them through the Softmax nonlinearity, $(a, b) \mapsto\left(\frac{1}{1+e^{b-a}}, \frac{1}{1+e^{a-b}}\right)$. The convolutional $A^{*}$ is trained using Adam SGD updates with learning rate $2^{-14}$ and weight decay of $2^{-3}$ ( $l_{2}$ parameter regularizer).

To simplify the task of the independent steganalyzer $A^{*}$ we use hand-crafted features instead of raw input vectors. These features are derived with the 8-bit reversal procedure and are strongly tailored to the LSB embedding. The main idea is that through "reversal" of the bit order in each element these features amplify the signal from the stego-embedding, which is naturally a weak high frequency distortion. Each value $v \in[-1,+1]$ in the input vector is transformed as follows:

- set $u=(v+1) \cdot \varepsilon$ for $\varepsilon=\frac{255}{2}$ and let $\hat{u}=\lfloor u\rfloor$

- convert $\hat{u}$ to an unsigned 8-bit value;

- $\operatorname{produce} \frac{1}{\varepsilon}(\operatorname{rev}(\hat{u})+(u-\hat{u}))-1$;

where $\operatorname{rev}(\cdot):\{0,1\}^{8} \mapsto\{0,1\}^{8}$ reverses the bit order: $\operatorname{rev}\left(\left(b_{0}, b_{1}, \cdots, b_{7}\right)\right)=\left(b_{7}, b_{6}, \cdots, b_{0}\right)$, with the bit at the first position in the sequence being the least significant bit (the LSB bit endianness). With these features any change in the least significant bit would affect the whole value on the $2^{7}$ scale instead of $2^{0}$, and make more prominent the arithmetic effects in the LSB matching due to \pm 1 addition.

\subsubsection{Results}

The performance of the Random Forest $A^{*}$ on the output vectors of the generator after $T$ iterations is shown in table 1. of the 1-d CNN - in Table 2. Both tables were obtained via the validation protocol and the feature preprocessing outlined above. It is worth noting that in both runs all metrics drop dramatically when the variety of embedded messages is increased, which lends evidence to the invariance of the induced distribution under the LSB distortion. Numerical experiments were also conducted on raw features, but both steganalyzers performed poorly.

We also experimented with the strength of the feedback to $G$ from the steganalyzer $A$ in SGAN training by setting $C_{\mathrm{tv}}=0$ and changing $C_{\mathrm{san}}$. The trained generator appears to be capable of successfully hiding message bits against the random forest $A^{*}$ even at the proportion $C_{1_{2}}: C_{\mathrm{san}}$ as low as $99: 1$. This can be attributed to simplicity of the generator's goal in this experiment. For $C_{\mathrm{san}}=10^{-3}$ and $C_{\mathrm{1}_{2}}=1-C_{\mathrm{san}}$, however, the generator failed to produce LSB-invariant output distribution even in the "Arbitrary" message generation scenario. This implies that the feedback provided by (4) is relevant to the task of generating cover vectors, which make the LSB matching embedding less susceptible to steganalysis.

The figures 6 and 7 show the performance dynamics of the tested steganalyzers. The generator converges to the desired output distribution is very quickly: in all SGAN re-runs with moderate to high values of $C_{\text {san }}$ the steganalyzer $A^{*}$ fails to discriminate between empty and non-empty vectors after at most 20 of training. Note that in the figures the proposed validation protocol was performed on each iteration during the first 100 SGAN iterations, and only on every 100-th iteration onward. 
Table 1: Performance metrics of the Random Forest $A^{*}$ on the cover vectors generated after all iterations of training $(\mu \pm 5 \sigma$ scaled by 100$)$.

\begin{tabular}{lcccc}
\hline $\begin{array}{l}\text { metric } \\
\text { type }\end{array}$ & ROC-AUC & F1-score & Accuracy & OOB \\
\hline Fixed & $100.0 \pm 0.0$ & $100.0 \pm 0.0$ & $100.0 \pm 0.0$ & 100.0 \\
& & & & \\
Pool (64) & $99.9 \pm 0.1$ & $99.9 \pm 0.2$ & $99.9 \pm 0.2$ & 99.9 \\
Pool (256) & $99.7 \pm 0.2$ & $98.9 \pm 0.6$ & $98.9 \pm 0.6$ & 98.8 \\
Pool (1024) & $84.7 \pm 3.0$ & $77.2 \pm 3.2$ & $76.7 \pm 2.9$ & 75.2 \\
Pool (4096) & $54.8 \pm 2.7$ & $53.1 \pm 3.8$ & $53.5 \pm 3.2$ & 52.4 \\
& & & & \\
Arbitrary & $49.6 \pm 3.0$ & $50.4 \pm 2.7$ & $49.8 \pm 2.2$ & 49.9 \\
\hline
\end{tabular}

Table 2: Performance metrics of the 1-d CNN $A^{*}$ on the cover vectors generated after all iterations of training $(\mu \pm 5 \sigma$ scaled by 100).

\begin{tabular}{lrrr}
\hline $\begin{array}{l}\text { metric } \\
\text { type }\end{array}$ & ROC-AUC & F1-score & Accuracy \\
\hline Fixed & $100.0 \pm 0.0$ & $99.9 \pm 0.1$ & $99.9 \pm 0.1$ \\
& & & \\
Pool (64) & $98.9 \pm 0.3$ & $95.6 \pm 1.3$ & $95.5 \pm 1.2$ \\
Pool (256) & $92.1 \pm 1.7$ & $84.7 \pm 2.6$ & $84.5 \pm 2.3$ \\
Pool (1024) & $70.0 \pm 4.5$ & $64.9 \pm 5.5$ & $64.6 \pm 3.7$ \\
Pool (4096) & $56.6 \pm 2.5$ & $55.1 \pm 4.8$ & $54.8 \pm 2.8$ \\
Arbitrary & $50.0 \pm 1.9$ & $49.9 \pm 5.9$ & $50.2 \pm 2.6$ \\
\hline
\end{tabular}

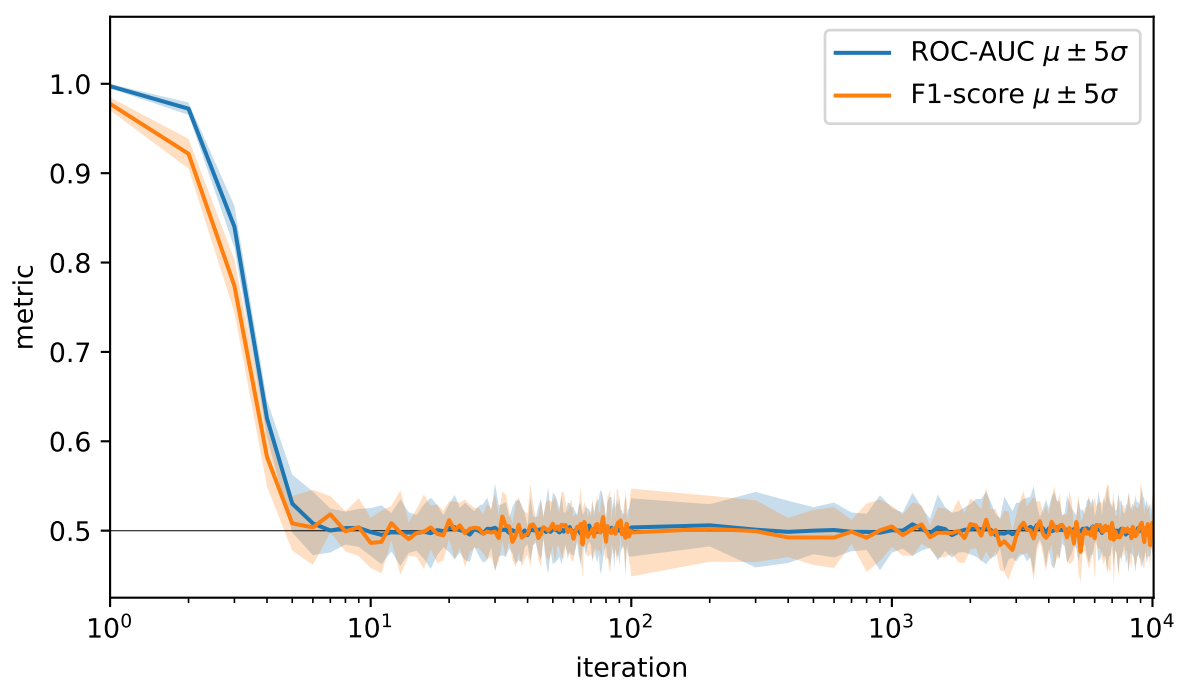

Figure 6: $k$-fold CV performance of $A^{*}$ (Random Forest) on synthetic images produced by the generator at different stages of training.

The main conclusion from this set of experiments is that the SGAN model (2) is useful in generating cover vectors, the unconditional distribution of which is invariant under the LSB embedding distortion (5). Also the embedding approximation (6) is adequate and provides relevant gradient feedback. In the next section we experiment with generating realistic cover images, which make the simple LSB embedding more steganographically secure. 


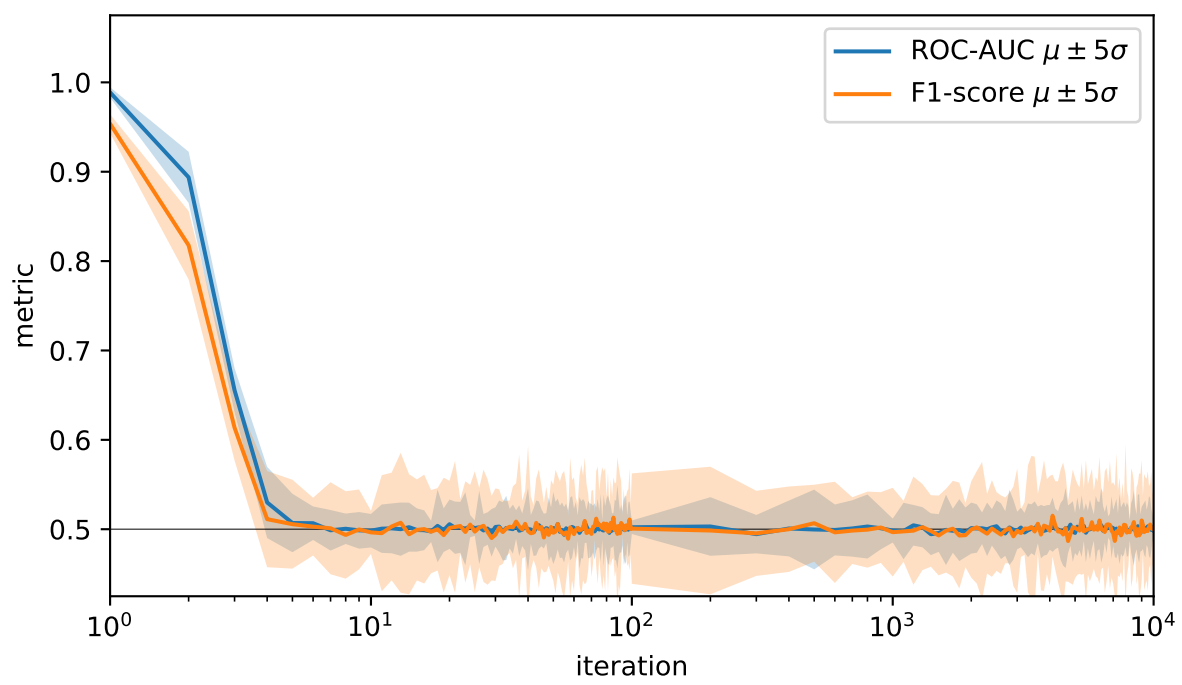

Figure 7: $k$-fold CV performance of $A^{*}(1-\mathrm{d} C N N)$ on synthetic images generated at different stages during the SGAN trainnig.

\subsection{Steganographic Images}

\subsubsection{Data Description}

In our experiments we use the Celebrities dataset [20] that contains 200000 images. All images were cropped to $64 \times 64$ pixels.

For steganalysis purposes we consider $10 \%$ of data as a test set. We denote the train set by $A$, the test set by $B$ and steganography algorithms used for hiding information by Stego $(x)$. After embedding some secret information we get the train set $A+\operatorname{Stego}(A)$ and the test set $B+\operatorname{Stego}(B)$, We end up with 380000 images for steganalysis training and 20000 for testing. For training the SGAN model we used all 200000 cropped images. After 8 epochs of training our SGAN produces images displayed in fig. 8

For information embedding we use the \pm 1 -embedding algorithm with a payload size equal to 0.4 bits per pixel for only one channel out of three. As a text for embedding we use randomly selected excerpts from some article from The New York Times.

\subsubsection{Experimental Setup}

In this section we describe the SGAN model structure. By $C 2 D-B N-L R$ we denote the following structural block of a convolutional neural network: Conv2d $\rightarrow$ Batch Normalization $\rightarrow$ Leaky ReLU.

The Steganalyser network $S$ and the Image Discriminator network have similar structure: four $C 2 D-B N-L R$ layers, then a fully connected layer (1 neuron) $\rightarrow$ Sigmoid function is used to compute an output. The Image generator network $G$ is (in order) a fully-connected layer (8192 neurons), four $C 2 D-B N-L R$ with Fractional-Strided convolution, then the Hyperbolic tangent function layer is used to compute normalised output.

The SGAN model is trained to solve (2) using the Adam optimization algorithm [15] with the learning rate $2^{-4}$ and update parameters $\beta_{1}=0.5$ and $\beta_{2}=0.999$. For each mini-batch of images we update weights of $D$ and $S$ once, then we update weights of $G$ twice.

In the following experiments, in addition to the steganalyser $S$ we use an independent steganalyser $S^{*}$. We define a filter $F^{(0)}$ that is special for steganalysis applications (see [26], [28], [30]), as follows

$$
F^{(0)}=\frac{1}{12}\left(\begin{array}{ccccc}
-1 & 2 & -2 & 2 & -1 \\
2 & -6 & 8 & -6 & 2 \\
-2 & 8 & -12 & 8 & -2 \\
2 & -6 & 8 & -6 & 2 \\
-1 & 2 & -2 & 2 & -1
\end{array}\right) \text {. }
$$




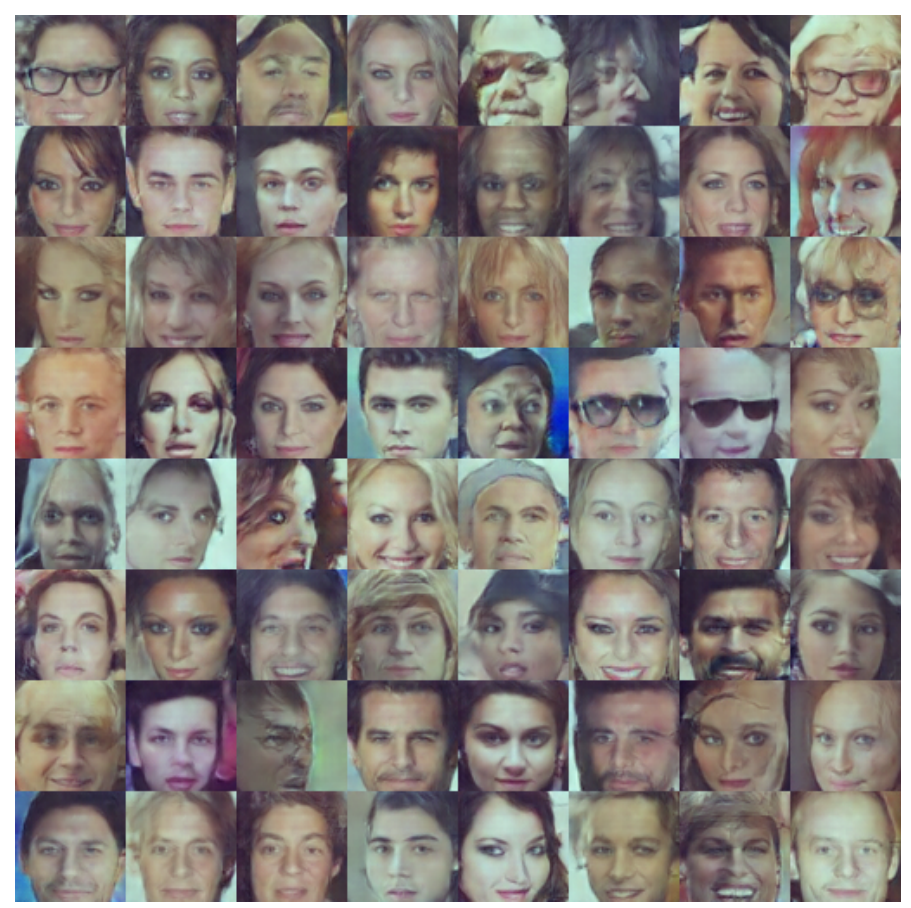

Figure 8: Examples of images, generated by SGAN after training for 8 epochs on the Celebrities dataset

Table 3: Accuracy of the steganalyser $S^{*}$ trained on real images

\begin{tabular}{|c|c|c|}
\hline Type of a test set $\backslash$ Image generator & SGANs & DCGANs \\
\hline Real images & \multicolumn{2}{|c|}{0.962} \\
\hline Generated images & 0.501 & 0.522 \\
\hline
\end{tabular}

The structure of the individual steganalyser $S^{*}$ has the form: 2D convolution with $F^{(0)}$ filter $\rightarrow$ Conv2D $\rightarrow$ Conv2D $\rightarrow$ Max Pooling $\rightarrow$ Conv2D $\rightarrow$ Conv2D $\rightarrow$ Max Pooling $\rightarrow$ Fully connected layer (1024 neurons) $\rightarrow$ Fully connected layer (1 neuron) $\rightarrow$ Sigmoid function for output. This structure provides state-of-the-art steganalysis accuracy, [26], and the filter $F^{(0)}$ allows to increase convergence speed of the steganalyser $S^{*}$ training.

For training of this steganalyser we use the Adam optimization algorithm on the loss $(2)$ with the learning rate equal to $5^{-6}, \beta_{1}=0.9, \beta_{2}=0.999$. As a loss function we use a binary cross-entropy.

The setup of experiments can be described as follows:

- We train and use the SGAN and/or DCGAN model to generate images to be used as containers;

- We train the independent steganalyser $S^{*}$ using either real images (sec.6.2.3) or generated images (sec. 6.2.4);

- We measure the accuracy of the steganalyser $S^{*}$.

\subsubsection{Training/Testing on Real Images}

In this set of experiments we train the independent steganalyser $S^{*}$ on real images. Results are provided in tab. 3 From the results we conclude that even the usual DCGAN generate synthetic container images, that can easily deceive a steganalyser. Although containers generated by on the SGAN model, which is specially designed to deceive the steganalyser, improve on the result of DCGAN, the improvement is not significant. These results also suggest that $S^{*}$ may have been overfit to the data distribution of the real images, and failed to generalize to the GAN approximation of the data distribution in the synthetic image domain.

Another interesting result follows from an experiment with HUGO steganography algorithm and SGAN generated containers:

- We use the \pm 1 -embedding algorithm for training the SGAN model; 
- We test the HUGO steganography analyzer on real images and on images, generated by the SGAN model.

Containers generated by the SGAN model decrease HUGO steganalysis accuracy from 0.624 to 0.499 , which demonstrates that the Steganographic Generative Adversarial Networks can potentially be used as a universal tool for generating Steganography containers tuned to deceive any specific steganalysis algorithm.

\subsubsection{Training/Testing on Generated Images}

In this set of experiments we train the steganalyser $S^{*}$ on images, generated using the DCGAN model from the prior noise distribution $p_{\text {noise }}(z)$ for some fixed seed value. In all experiments the size of the train set is fixed at $\approx 160000$. After training we test the analyser $S^{*}$ on images generated with the DCGAN model according to the following experimental setups:

C1. We use the same seed value;

C1. We use the same seed value, as in C1, and we additionally tune the DCGAN model for several epochs.

Table 4: Training/testing on generated images according to experimental conditions C1-C3

\begin{tabular}{|c|c|}
\hline Experimental Conditions & Accuracy \\
\hline C1 & 0.982 \\
\hline C3 & 0.499 \\
\hline
\end{tabular}

The experiment results in tab. 4 indicate that additional tuning of the image generator $G$ make this effect even more pronounced.

We also conduct an experiment with classification of generated images without steganographic embeddings. For this purposes we train a DCGAN conditional model on the MNIST dataset, and train a separate classifier for the MNIST classification task. The trained classifier achieved almost perfect accuracy both on the held-out real MNIST dataset, and on synthetic images produced by the DCGAN. This provides evidence that it is possible to train an image classifier that shows acceptable accuracy both on real and synthetic images. However it is the artificial generation of image containers that breaks the usual approaches to steganalysis.

\section{INFORMATION ENCRYPTION WITH SEGAN}

\subsection{Data description}

For the experiments in the current section we use MNIST [19] and CIFAR-10 [18] data sets. Both datasets can be considered as a benchmark in Deep Learning and Compute Vision.

- The MNIST dataset is a set of gray handwritten digits from 0 to 9 of size $28 \times 28$;

- The CIFAR-10 dataset consists of $6000032 \times 32$ RGB images of totally 10 classes: airplane, automobile, bird, cat, deer, dog, frog, horse, ship, truck.

\subsection{Encryption experiments}

We consider SEGAN model as a model for information encryption. It allows to generate images with hidden information inside. In the experimental section we should

- check if the generated images are realistic;

- look at the quality of encryption.

The examples of generate images for MNIST dataset are presented in Fig. 9 We observe the full realism in the generated images.

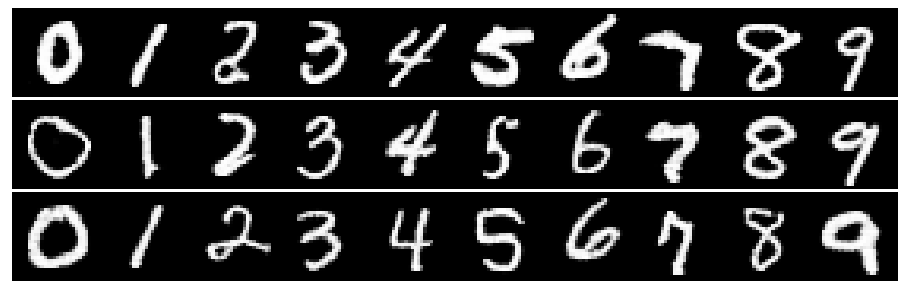

Figure 9: Sample synthetic images: MNIST 
The examples of generate images for CIFAR dataset are presented in Fig. 10 This images are small and looks quit realistic. This level of realism occurs because of current limitation of generative modeling.

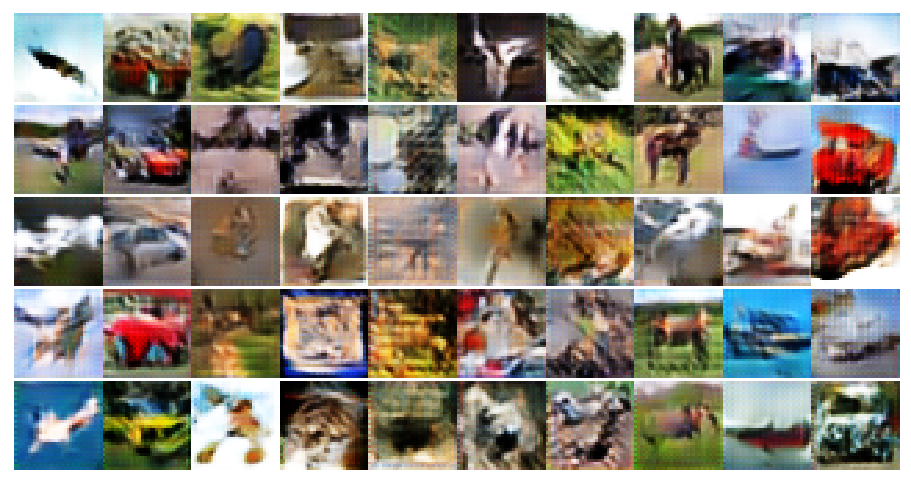

Figure 10: Sample synthetic images: CIFAR-10

The quality of encryption is presented in Table 5 . As a measure of quality we considered

$$
\mathbb{E}_{M}\left[\#\left\{i: M_{i} \neq B\left(A\left(M_{i}\right)\right)\right\}\right],
$$

which means the average percentage of reconstructed pixels. As we can see, our new model allows to encrypt-decrypt messages with the quality of almost 1 . It is a little bit harder to encrypt longer messages that short.

Table 5: Quality of encryption/decryption (\% of reconstruction bits)

\begin{tabular}{|c|c|c|c|}
\hline Dataset INumber of bits & $\mathbf{1 6}$ bit & $\mathbf{3 2}$ bit & 64 bit \\
\hline MNIST & 99.98 & 99.54 & 98.65 \\
\hline CIFAR-10 & 99.96 & 99.82 & 99.81 \\
\hline
\end{tabular}

\section{CONCLUSIONS}

In this work,

1. We open a new field for applications of Generative Adversarial Networks, namely, container generation for steganography applications;

2. We consider the \pm 1 -embedding algorithm and test novel approaches to more steganalysis-secure information embedding: we demonstrate that both SGAN and DCGAN models are capable of decreasing the detection accuracy of a steganalysis method almost to that of a random classifier;

3. A model for secure adaptive steganographic containers generation has been presented;

4. A number of ways to adopt in order to deceive steganalysis has been proposed;

5. A new GAN-based steganography model has been proposed and tested on MNIST and CIFAR-10 dataset;

6. As a result an article ([31]) has been written and submitted to NIPS 2016, Workshop on Adversarial Training. This paper cross-checks and significantly extends results of that initial paper.

\section{References}

[1] Abbas Cheddad, Joan Condell, Kevin Curran, and Paul Mc Kevitt. Digital image steganography: Survey and analysis of current methods. Signal Processing, 90(3):727 - 752, 2010.

[2] Ishan Durugkar, Ian Gemp, and Sridhar Mahadevan. Generative multi-adversarial networks. arXiv preprint arXiv:1611.01673, 2016.

[3] Tomáš Filler, Jan Judas, and Jessica Fridrich. Minimizing additive distortion in steganography using syndrome-trellis codes. IEEE Transactions on Information Forensics and Security, 6(3):920-935, Sept 2011.

[4] Jessica Fridrich and Jan Kodovskỳ. Rich models for steganalysis of digital images. IEEE Transactions on Information Forensics and Security, 7(3):868-882, 2012.

[5] Yaroslav Ganin, Evgeniya Ustinova, Hana Ajakan, Pascal Germain, Hugo Larochelle, François Laviolette, Mario Marchand, and Victor Lempitsky. Domain-adversarial training of neural networks. ArXiv e-prints, May 2015. 
[6] Ian Goodfellow. Nips 2016 tutorial: Generative adversarial networks. ArXiv e-prints, December 2017.

[7] Ian Goodfellow, Jean Pouget-Abadie, Mehdi Mirza, Bing Xu, David Warde-Farley, Sherjil Ozair, Aaron Courville, and Yoshua Bengio. Generative adversarial nets. pages 2672-2680, 2014.

[8] Martin Heusel, Hubert Ramsauer, Thomas Unterthiner, Bernhard Nessler, Günter Klambauer, and Sepp Hochreiter. Gans trained by a two time-scale update rule converge to a nash equilibrium. ArXiv e-prints, June.

[9] Vojtěch Holub and Jessica Fridrich. Designing steganographic distortion using directional filters. In WIFS, 2012.

[10] Vojtěch Holub, Jessica Fridrich, and Tomáš Denemark. Universal distortion function for steganography in an arbitrary domain. EURASIP Journal on Information Security, 2014(1):1-13, 2014.

[11] Zhiting Hu, Zichao Yang, Ruslan Salakhutdinov, and Eric P. Xing. On unifying deep generative models. ArXiv e-prints, June 2017.

[12] Sergey Ioffe and Christian Szegedy. Batch normalization: Accelerating deep network training by reducing internal covariate shift. arXiv preprint arXiv:1502.03167, 2015.

[13] Phillip Isola, Jun-Yan Zhu, Tinghui Zhou, and Alexei A. Efros. Image-to-image translation with conditional adversarial networks. ArXiv e-prints, November 2016.

[14] Diederik Kingma and Jimmy Ba. Adam: A method for stochastic optimization. arXiv preprint arXiv:1412.6980, 2014.

[15] Diederik Kingma and Jimmy Ba. Adam: A method for stochastic optimization. arXiv preprint arXiv:1412.6980, 2014.

[16] Diederik P. Kingma, Danilo J. Rezende, Shakir Mohamed, and Max Welling. Semi-supervised learning with deep generative models. ArXiv e-prints, June 2014.

[17] Diederik P. Kingma and Max Welling. Auto-encoding variational bayes. ArXiv e-prints, December 2013.

[18] Alex Krizhevsky and G Hinton. Convolutional deep belief networks on cifar-10. Unpublished manuscript, $40,2010$.

[19] Yann LeCun. The mnist database of handwritten digits. http://yann. lecun. com/exdb/mnist/, 1998.

[20] Ziwei Liu, Ping Luo, Xiaogang Wang, and Xiaoou Tang. Deep learning face attributes in the wild. In Proceedings of International Conference on Computer Vision (ICCV), 2015.

[21] Chandreyee Maiti, Debanjana Baksi, Ipsita Zamider, Pinky Gorai, and Dakshina Ranjan Kisku. Data Hiding in Images Using Some Efficient Steganography Techniques, pages 195-203. Springer Berlin Heidelberg, Berlin, Heidelberg, 2011.

[22] Sebastian Nowozin, Botond Cseke, and Ryota Tomioka. $f$-gan: Training generative neural samplers using variational divergence minimization. ArXiv e-prints, June 2016.

[23] Tomás Pevnỳ, Patrick Bas, and Jessica Fridrich. Steganalysis by subtractive pixel adjacency matrix. IEEE Transactions on Information Forensics and Security, 5(2):215-224, 2010.

[24] Tomáš Pevný, Tomáš Filler, and Patrick Bas. Using high-dimensional image models to perform highly undetectable steganography. In Information Hiding, page 2010, Calgary, Canada, June 2010.

[25] Lionel Pibre, Pasquet Jérôme, Dino Ienco, and Marc Chaumont. Deep learning for steganalysis is better than a rich model with an ensemble classifier, and is natively robust to the cover source-mismatch. ArXiv e-prints, November 2015.

[26] Lionel Pibre, Pasquet Jérôme, Dino Ienco, and Marc Chaumont. Deep learning for steganalysis is better than a rich model with an ensemble classifier, and is natively robust to the cover source-mismatch. arXiv preprint arXiv:1511.04855, 2015.

[27] Yinlong Qian, Jing Dong, Wei Wang, and Tieniu Tan. Deep learning for steganalysis via convolutional neural networks. In SPIE/IS\&T Electronic Imaging, pages 94090J-94090J. International Society for Optics and Photonics, 2015.

[28] Yinlong Qian, Jing Dong, Wei Wang, and Tieniu Tan. Deep learning for steganalysis via convolutional neural networks. In IS\&T/SPIE Electronic Imaging, pages 94090J-94090J. International Society for Optics and Photonics, 2015.

[29] Alec Radford, Luke Metz, and Soumith Chintala. Unsupervised representation learning with deep convolutional generative adversarial networks. arXiv preprint arXiv:1511.06434, 2015.

[30] Shunquan Tan and Bin Li. Stacked convolutional auto-encoders for steganalysis of digital images. In Asia-Pacific Signal and Information Processing Association, 2014 Annual Summit and Conference (APSIPA), pages 1-4. IEEE, 2014.

[31] Denis Volkhonskiy, Ivan Nazarov, Boris Borisenko, and Evgeny Burnaev. Steganographic generative adversarial networks. Workshop on Adversarial Training, Neural Information Processing Systems, 2016. 